\title{
Review Article \\ The Mitochondrial Aminoacyl tRNA Synthetases: Genes and Syndromes
}

\author{
Daria Diodato, Daniele Ghezzi, and Valeria Tiranti \\ Unit of Molecular Neurogenetics, Pierfranco and Luisa Mariani Center for the Study of Mitochondrial Disorders in Children, IRCCS \\ Foundation Neurological Institute "C. Besta", Via Temolo 4, 20126 Milan, Italy
}

Correspondence should be addressed to Valeria Tiranti; valeria.tiranti@istituto-besta.it

Received 13 September 2013; Accepted 1 December 2013; Published 4 February 2014

Academic Editor: R. Seger

Copyright (C) 2014 Daria Diodato et al. This is an open access article distributed under the Creative Commons Attribution License, which permits unrestricted use, distribution, and reproduction in any medium, provided the original work is properly cited.

Mitochondrial respiratory chain (RC) disorders are a group of genetically and clinically heterogeneous diseases. This is because protein components of the RC are encoded by both mitochondrial and nuclear genomes and are essential in all cells. In addition, the biogenesis and maintenance of mitochondria, including mitochondrial DNA (mtDNA) replication, transcription, and translation, require nuclear-encoded genes. In the past decade, a growing number of syndromes associated with dysfunction of mtDNA translation have been reported. This paper reviews the current knowledge of mutations affecting mitochondrial aminoacyl tRNAs synthetases and their role in the pathogenic mechanisms underlying the different clinical presentations.

\section{Introduction}

Mitochondria are double-membrane cytoplasmic organelles essential for energy supply to the cell. Adenosine- $5^{\prime}$ triphosphate (ATP), the molecular unit of currency for intracellular energy transfer, is produced by the last of five multisubunit complexes embedded in the inner mitochondrial membrane, which form the respiratory chain (RC) responsible for oxidative phosphorylation (OXPHOS).

Mitochondria have their own DNA (mtDNA) that is a circular, double-stranded molecule; in humans, it is 16.569 base pairs long and contains genes encoding 13 protein subunits of RC complexes I, III, IV, and V as well as transfer ( $t$ ) and ribosomal (r) RNA encoding genes for mtDNA-specific translation. However, hundreds of additional gene products, providing the components necessary for mtDNA replication and expression, many RC complex subunits, and the complex protein network needed for RC formation, activity, and turnover, are nuclear-encoded.

The term mitochondrial disorder refers to diseases that are caused by OXPHOS dysfunction and comprises a clinically and genetically heterogeneous group of syndromes that all together are amongst the most common inherited human diseases, with a prevalence of $1: 5000$.

Deficiencies of single RC complex are generally caused by mutations in genes encoding structural subunits or proteins involved in the assembly of a specific OXPHOS enzyme complex. On the contrary, combined OXPHOS defects are often associated with impairment of processes such as replication, transcription, or translation of mtDNA, which can be due to mutations in either mtDNA-encoded RNAs (tRNAs and rRNAs) or nuclear DNA-encoded proteins $[1,2]$. Hundreds of RNA and protein factors have been maintained through evolution of eukaryotes to carry out the synthesis of a few, but essential, mtDNA-encoded proteins, carried out in situ by organelle-specific translation machinery [3].

Given the multitude of proteins required for proper mitochondrial protein synthesis, it is not surprising that mitochondrial disorders due to impairment of this essential process are genetically heterogeneous and that, in many patients, the molecular genetic defect remains unknown.

This review will focus on a group of enzymes with a key role in mitochondrial protein synthesis, the aminoacyl tRNA synthetases (mt-aaRSs), mutations of which are responsible 
for an increasing number of OXPHOS deficiencies and diseases (Table 1).

\section{Mitochondrial Protein Synthesis}

Protein synthesis is a complex process that in mitochondria supplies the mtDNA-encoded subunits of RC complexes through an organellar-specific translational apparatus distinct from the cytosolic counterpart. In fact, there are some distinguishing features for the mitochondrial translation, including the mitochondrial genetic code, which differs from the universal one (i.e., AUA codes for methionine and UGA, instead of being a stop codon, codes for tryptophan), and the structure of mitochondrial mRNAs, which have no or few $5^{\prime}$ untranslated nucleotides, is uncapped and contains a poly (A) immediately after the stop codon.

The human mitoribosome is made up of 2 ribosomal RNAs (rRNA) and 81 mitochondrial ribosomal proteins (MRPs) and comprises two subunits, the small (SSU or 28S) and the large (LSU or 39S) [3]. Mammalian mitoribosome differs markedly from bacteria, cytosolic, and also from other mitochondrial ribosomes, due to the peculiar recruitment of numerous extraproteins. Most of the protein components involved in mitochondrial translation such as a multitude of initiation, elongation and termination factors, subunits and assembly protein of mitoribosome, tRNA-modifying enzymes, and the mt-aaRSs are encoded by nuclear genes, whereas 2 mitochondrial ribosomal and 22 transfer RNAs (rRNAs and tRNAs) are encoded by mtDNA [4]. Mitochondrial mRNA translation has been hypothesized to take place in a complex bound to the inner membrane through electrostatic force and maybe protein interaction [5]. The basic model of mitochondrial protein synthesis is derived from studies in bacteria and can be divided into three phases: initiation, elongation, and termination $[3,6]$.

Due to the unusual characteristics of mitochondrial mRNA, it is not fully clear how the protein synthesis starts. It is thought that mitochondrial initiation factor mtIF3 (the ortholog of prokaryotic IF3) induces the dissociation of the mitoribosome into its two components, the small (SSU or 28S) and the large (LSU or 39S) subunits. This facilitates the binding of the mRNA to the SSU subunit directing the ribosome to the start codon. Mammalian mitochondria use a single tRNA ${ }^{\text {Met }}$ for both the initiation and elongation phases; after aminoacylation, the $\mathrm{tRNA}^{\mathrm{Met}}$ needs to be formylated by methionyl-tRNA transformylase (forming fMet-tRNA $^{\text {Met }}$ ) to initiate mitochondrial translation. A second mitochondrial initiation factor, mtIF2, is required for binding of the fMettRNA $^{\text {Met }}$ to the SSU. Recombining of the mitoribosome subunits and GTP hydrolysis promote the release of mtIF3 and mtIF2 and the completion of the initiation phase.

In the elongation phase, a crucial role is played by the elongations factors mtEFTu, mtEFTs, and mtEFG (1 and 2). mtEFTu forms a complex with GTP and a tRNA charged with its corresponding amino acid, protecting the latter from hydrolysis and facilitating the codon-anticodon recognition at the acceptor site A of the SSU. This step requires GTP hydrolysis and leads to the release of mtEFTu. The aminoacyltRNA moves into the peptidyl (P) site of the mitoribosome, where the amino acid is added to the growing peptide. mtETFs recycles the released mtEFTu-GDP, reforming an active mtEFTu-GTP. mtEFG1 catalyzes the translocation of tRNAs from $P$ to exit (E) site of the mitoribosome, while mRNA is advanced by one codon. This last step has been derived from the prokaryotic model; the $\mathrm{E}$ site has been suggested to be very weak or even absent in the mitoribosome. Finally, the tRNA leaves the mitoribosome and a new elongation cycle can start. The functional role of mtEFG2 in mitochondrial translation is not completely understood.

The termination phase initiates with the recognition of a stop codon by the mitochondrial release factor (mtRF1 or mtRF1a). This causes the detachment of the polypeptide linked to the last tRNA present in the P site. After release of the newly synthesized protein, mitochondrial ribosome recycling factors ( $m$ tRRF and possibly $m t E F G 2$ ) enable the mitoribosomal subunits, tRNA and mRNA, to dissociate from each other, allowing the reuse of them for a new round of protein synthesis $[7,8]$.

Mitochondrial disorders with different clinical presentation have been associated with defects in several enzymes involved in mitochondrial translation including tRNA-modifying enzyme like TRMU (tRNA 5-methylaminomethyl-2-thiouridylate methyltransferase, OMIM \#613070) [9] and MTO1 (Mitochondrial Translation Optimization 1, OMIM\#614702) [10] or elongation factors like GFM1/EFG1 (OMIM\#609060), TSFM/EFTs (OMIM \#610505), and TUFM/EFTu (OMIM\#610678) [11, 12].

\section{Aminoacyl-tRNA Synthetases}

Aminoacyl-tRNA synthetases (aaRSs) are key enzymes in the translation of the genetic information. In fact, they catalyze the specific attachment of each of the 20 amino acids (aa) to a cognate tRNA, through a two-step reaction, where they first activate the amino acid with ATP, forming an intermediate aminoacyl-adenylate, and then transfer the aminoacyl group to the $3^{\prime}$-end of its own tRNA $[3,13]$.

With the exception of GARS and KARS, mitochondrial and cytoplasmic aaRSs are encoded by distinct nuclear genes. The gene for mitochondrial glutaminyl-tRNA synthetase has not been found yet, possibly because mitochondrial glutamyltRNA synthetase (EARS2) efficiently misaminoacylates $\mathrm{mt}$ $\mathrm{tRNA}^{\text {Gln }}$ to form glutamate charged-tRNA ${ }^{\text {Gln }}$ [14]. Hence, mt-aaRSs are 19 instead of 20, including the 2 with double localization.

Mt-aaRSs are encoded by nuclear genes (aaRS2), imported into mitochondria, and their interaction with cognate tRNAs seems to be essential for their amount and stability [15]. Mt-tRNA genes are located in three transcription units: the short $\mathrm{H}$-strand unit (with the rRNA region and two tRNA genes) is transcribed twice as fast as the L-strand that contains eight tRNA genes and more frequently then the entire $\mathrm{H}$-strand unit that produces 14 tRNAs. King and Attardi showed that steady-state levels of 


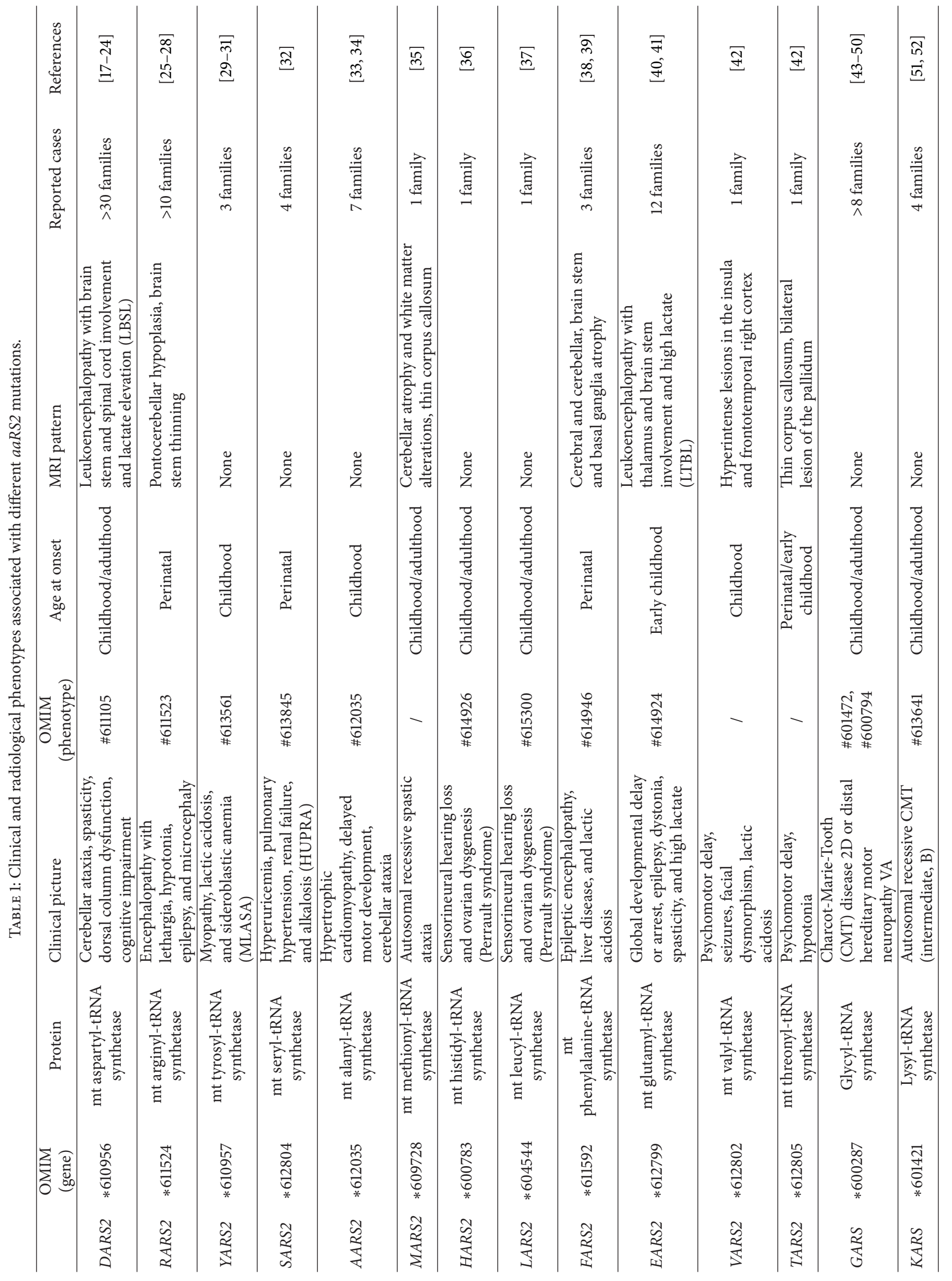


different tRNAs remain uniform suggesting a mechanism of posttranscriptional regulation of redundant tRNAs [16].

aaRSs structure typically presents a catalytic domain and an anticodon binding domain; some of them have also an editing domain to deacylate mischarged amino acids, preventing insertion of incorrect amino acids during protein synthesis [17]. These enzymes can be classified into two distinct classes according to the structure of the catalytic site: class I enzymes bear the classical Rossmann fold (defined as an adenylic-nucleotides recognition site) that displays five parallel $\beta$-strands connected via $\alpha$-helices and the two signature motifs; class II enzymes display an alternate folding, mainly constituted by a sheet of six antiparallel $\beta$-strands and three motifs of less-conserved sequences [18].

During evolution, aaRSs have acquired additional domains and insertions thus expanding their range of function. Cytosolic aaRSs have been discovered to play many other roles with either extracellular (cytokines or angiogenesis regulators) or cytoplasmic activities being involved in apoptosis, synthesis of rRNA, or tRNA export to the cytosol. On the other hand, also mt-aaRSs have been investigated for having additional functions; for instance, it has been demonstrated that the yeast homologue of human mitochondrial lysyl-tRNA synthetase is a dual function protein, involved in both aminoacylation of mitochondrial tRNA ${ }^{\text {Lys }}$ and import of cytosolic tRNA ${ }^{\text {Lys }}$ into mitochondria[19].

\section{Aminoacyl-tRNA Synthetases and Mitochondrial Disorders}

An increasing fraction of mitochondrial protein synthesis deficiencies are caused by mutations in one of the aaRS2 genes and have been associated with diverse clinical presentations, usually with an early onset and transmitted as autosomal recessive traits. Such a broad clinical spectrum can be partially explained by the multiple and still unknown functions of mt-aaRSs. However, there is a strict genotypephenotype correlation for most of these genes, albeit the reason for specific and different cellular or tissue damage, being all aaRSs ubiquitous enzymes working in the same pathway is not clear. Because of the fundamental function of these enzymes, it has been suggested that mt-aaRSs loss of function mutations is not compatible with extrauterine life and that a residual enzymatic activity can result in different tissue alterations after development [20].

We present an overview of the main clinical presentations associated with mutations in aaRS $2 s$, which have been identified in the last 10 years, initially by linkage mapping/homozygosity mapping and candidate gene analysis and, more recently, by next-generation whole exome sequencing (WES).

4.1. DARS2. DARS2, encoding the mitochondrial aspartyltRNA synthetase (mt-AspRS), was the first aaRS2 gene reported to cause a human disease, characterized by a peculiar leukoencephalopathy named LBSL (leukoencephalopathy with brain stem and spinal cord involvement, OMIM
\#611105) [21] associated with cerebellar ataxia, spasticity, and variable degree of cognitive impairment. To date, more than 30 families have been described with mutations in DARS2 (Table 1). In mutant patients, high lactate has been observed only in the affected white matter. White matter changes, involving brain stem and spinal cord tracts, are very peculiar. Almost all patients with LBSL are compound heterozygotes, sharing a complex rearrangement in one allele that involves a T-C stretch upstream from exon 3 (228-20/-21delTTinsC) and a second variable, usually missense, mutation. The splice site mutation in intron 2 is a hypomorphic mutation that partially interferes with the splicing of exon 3, leading to frameshift and premature truncation (Arg76SerfsX5) of only a fraction of DARS2 transcripts, maintaining some mt-AspRS residual activity. Van Berge et al. tried to explain the brain tissue specificity showing differences in mRNA splicing process between neurons and other cells [22]. Some compound heterozygous patients have been reported to show atypical presentations including specific MRI pattern associated with no clinical symptoms [23] or no lactate elevation [24]. Axonal neuropathy was an important and frequent feature of LBSL [25]. Homozygous DARS2 mutations have been shown to cause a more severe phenotype $[26,27]$ or exercise-induced paroxysmal gait ataxia and areflexia [28].

Surprisingly, blue-native PAGE as well as spectrophotometric measurements often revealed normal OXPHOS enzyme activities in muscle and cultured cells.

4.2. RARS2. Mutations in RARS2 gene have been described so far in more than 10 families (Table 1). The first patients described with mutations in RARS2, the gene encoding for the mitochondrial arginyl-tRNA synthetase (mt-ArgRS), presented progressive atrophy in cerebellum, pons (pontocerebellar hypoplasia type 6, PCH6; OMIM\#611523), with cerebral cortex and white matter involvement, causing a severe infantile clinical picture characterized by lethargy, seizures with apneic spells, hypotonia, and premature death. These consanguineous Sephardic Jewish patients harboured a homozygous splice site variant (IVS2 +5A>G) [29] and showed multiple RC defects in muscle and fibroblasts. However, at least three RARS2 mutant patients, carrying missense mutations, have been reported without any $\mathrm{RC}$ defect in muscle [30, 31]. Later, it has been demonstrated that the subtype 1 of pontocerebellar hypoplasia (OMIM\#607596), characterized by a neuropathological profile with loss of spinal anterior horn cells, diffuse gliosis, flat cerebellar folia, loss of Purkinje cells, and pontocerebellar hypoplasia, can be also caused by mutation in RARS2 [32]. Recently, compound heterozygous RARS2 mutations have been described in patients with neonatal onset epileptic encephalopathy and typical PCH6 MRI findings. These mutations have been shown to cause reduced RARS2 amount and activity, associated with impaired tRNA ${ }^{\text {Arg }}$ stability [31].

4.3. YARS2. YARS2 encodes for the mitochondrial tyrosyltRNA synthetase (mt-TyrRS). Only two missense mutations in this gene have been reported till now, associated with myopathy, lactic acidosis, and sideroblastic anemia (MLASA, 
OMIM\#613561). These mutations, present in homozygous state in different patients, affect the enzyme catalytic domain causing generalized mitochondrial translation defect in myoblasts [33]. Recently, a more severe phenotype has been described associated with the known homozygous mutation c.156C>G; p.Phe52Leu [34]. Complexes I, III, and IV were defective in skeletal muscle but not in fibroblasts. Gel filtration experiments showed that mt-TyrRS is part of high-molecular-weight complexes ( 250 kDa and $1 \mathrm{MDa})$, suggesting that, like some of the cytoplasmic aaRSs, mt-aaRSs could also occur in high-molecular-weight complexes [35].

4.4. SARS2. Belostotsky et al. [36] described three probably related Palestinian infants with a homozygous mutation c.1169A $>$ G (p.Asp390Gly) in SARS2, the gene encoding the mitochondrial seryl-tRNA synthetase (mt-SerRS). They were affected by a multisystem disorder, characterized by prematurity, progressive renal failure leading to electrolyte imbalances, metabolic alkalosis, and pulmonary hypertension, called HUPRA syndrome (Hyperuricemia, pulmonary hypertension, renal failure, and alkalosis, OMIM\#613845), and died within the first 14 months of life. Global developmental delay was an additional feature.

Mt-tRNA $^{\text {Ser }}$ catalyzes the ligation of serine to two mitochondrial tRNA isoacceptors: tRNA ${ }^{\text {Ser(AGY) and }}$ tRNA $^{\text {Ser(UCN) }}$. The Asp390Gly mutation impacts the acylation of tRNA $^{\text {Ser(AGY) }}$ but probably not that of tRNA ${ }^{\text {Ser(UCN) }}$ with severe decrease in the expression of the nonacylated transcript and the complete absence of the acylated tRNA ${ }^{\text {Ser(AGY) }}$.

Recently, a novel SARS2 homozygous mutation c.1205G >A (p.Arg402His) has been identified in a girl and her brother with HUPRA syndrome [37].

4.5. AARS2. AARS2 encodes the mitochondrial alanyl-tRNA synthetase (mt-AlaRS). All alanyl-tRNA synthetase orthologs have an aa binding pocket equally accessible by alanine, serine, and glycine; hence, this enzyme contains an editing domain, in addition to the catalytic one, essential to deacylate the mischarged tRNAs. In 2011, Götz et al. [38] described $A A R S 2$ mutations causing hypertrophic cardiomyopathy and lactic acidosis in infants from two different families, one being homozygous for the p.Arg592Trp mutation and the other being compound heterozygous for the p.Arg592Trp and the missense p.Leu155Arg. All patients died at few months of age; they showed a drastic deficiency of RC complexes I, III, and IV in heart tissue, with less severe defects in brain and skeletal muscle (OMIM\#612035). Patients' fibroblasts or myoblasts did not show any OXPHOS defect. p.Arg592Trp localizes in the editing domain, thus probably affecting the recognition of mischarged tRNAs, while the other missense mutation is located in the aminoacylation domain.

AARS2 mutations can be also associated with a different clinical presentation, characterized by cerebellar ataxia and psychotic features with leukoencephalopathy [39].

4.6. MARS2. Bayat et al. [40] described mutations in MARS2 encoding the mitochondrial methionyl-tRNA synthetase
(mt-MetRS), which cause autosomal recessive spastic ataxia with leukoencephalopathy (ARSAL or spastic ataxia 3, OMIM\#611390) in humans and neurodegeneration in flies. Patients' brain MRI showed cerebellar atrophy and white matter alterations sometimes associated with thin corpus callosum; disease onset was very variable. In all ARSAL subjects, complex gene rearrangements were identified in $M A R S 2$, including homozygous duplication or compound heterozygous duplication-deletion (in this last case, disease onset was anticipated). Despite increased levels of aberrant MARS2 transcripts, protein levels were lower in all patients. Furthermore, patients' fibroblasts displayed a reduced complex I activity as in mutant flies and immortalized lymphoblast lines showed an impaired translation [40].

4.7. HARS2. Mutations in HARS2, encoding the mitochondrial histidyl-tRNA synthetase (mt-HisRS), have been associated with Perrault syndrome in a family with five members affected (OMIM\#614926). Perrault syndrome is a clinical entity characterized by ovarian dysgenesis and sensorineural hearing loss. This recessive syndrome is genetically heterogeneous, being caused by mutations in different genes (HSD17B4, CLPP, and LARS2, besides HARS2), and is associated with premature ovarian failure in females and progressive hearing loss in both males and females. The HARS2 mutant patients were compound heterozygous for two missense mutations, p.Val368Leu and p.Leu200Val, the latter creating an alternative splice site that causes an inframe deletion of 12 codons. Both missense mutations caused significantly decreased enzyme activity compared to wild type. The enzymatic defect and the oxidative phenotype in the yeast model were more severe for the p.Val368Leu mutation [41].

4.8. LARS2. More recently homozygous mutations in LARS2, encoding the mitochondrial leucyl-tRNA synthetase (mtLeuRS), have been found in patients with Perrault syndrome (OMIM\#615300). Three affected subjects from a consanguineous Palestinian family carried a homozygous p.Thr522Asn substitution of a highly conserved residue in the catalytic domain, whereas compound heterozygous mutations were found in a Slovenian woman [42]: a c.1866C>T transition resulting in a p.Thr629Met substitution at a residue conserved in mammals, and a 1-bp deletion (c.1077delT) predicted to yield a 373-amino-acid truncated protein with 14 novel amino acids at the C terminus (p.Ile360PhefsTer15). Analysis in yeast models suggested reduced activity relative to wild type for the identified mutations [42].

4.9. FARS2. Mutations in the mitochondrial phenylalaninetRNA synthetase, FARS2 (mt-PheRS), have been identified by WES in two couples of siblings, characterized by fatal epileptic encephalopathy, liver disease, and lactic acidosis. Neuropathological findings together with liver disease filled the criteria for Alpers-Huttenlocher syndrome (OMIM\#614946). All FARS2 mutant patients died within the first two years of life. The Saudi siblings were homozygous for a homozygous 431A-G transition, resulting in a Tyr144Cys (Y144C) 
substitution at a highly conserved residue in the catalytic domain [43]. The Finnish siblings were compound heterozygous for two missense variants, c.986T $>C$ (p.Ile329Thr) and c.1172A $>$ T (p.Asp391Val), affecting residues in the ATPbinding site in the aminoacylation domain, or in the anticodon stem-binding domain, respectively [44]. Blue-native gel electrophoresis showed a defective complex IV in both brain and muscle, whereas complex I was decreased only in brain. Patients' fibroblast displayed neither biochemical defects nor impaired mitochondrial translation [44].

Recently, a partial genomic deletion and a p.Asp325Tyr missense mutation in FARS2 have been described in a patient with early-onset epilepsy and isolated complex IV deficiency in muscle. The biochemical defect was found in myoblasts but not in fibroblasts and was associated with decreased steadystate levels of COXI and COXII proteins and reduced steadystate levels of the mt-tRNA ${ }^{\text {Phe }}$ transcript [45].

4.10. EARS2. EARS2 encodes for the mitochondrial glutamyl-tRNA synthetase (mtGluRS). Mutations in this gene have been identified by WES in a single Italian patient and then found in 11 additional patients with similar MRI alterations, described as LTBL (leukoencephalopathy with thalamus and brain stem involvement and high lactate, OMIM\#614924) [46]. Only one additional patient with EARS2 mutations has been described to date [47]. EARS2 mutant patients presented a peculiar biphasic clinical course: an infantile disease onset with rapid progression followed by stabilization, and in some cases improvement, and partial recovery of lost skills. MRI displayed abnormal thalami, midbrain, pons, and medulla oblongata and alterations of cerebral and cerebellar white matter. High blood lactate and OXPHOS defects in muscle and fibroblasts were present. All patients were compound heterozygous for a severe mutation and a milder one, suggesting that there is a threshold for mtGluRS activity to cause the disease. The only patient reported with a homozygous severe mutation (p.Lys65Glu) presented a dramatic phenotype with brain and liver involvement and a fatal outcome at age 3 months [47].

4.11. VARS2 and TARS2. Recently, mutations in other two $\mathrm{mt}$-aaRSs have been reported as causative of severe recessive infantile disorders with OXPHOS deficiency. A homozygous missense mutation (p.Thr367Ile) in VARS2, the gene encoding the mitochondrial valyl-tRNA synthetase (mt-ValRS), has been found in a child with psychomotor delay, seizures, and facial dysmorphisms. Two siblings with phenotype characterized by hypotonia, psychomotor retardation, and premature death were found to be compound heterozygous for a splice site (c.695 +3A >G) and a missense (p.Pro282Leu) mutation in the mitochondrial threonyl-tRNA synthetase gene TARS2 [39].

4.12. GARS and KARS. In addition to genes coding for mitochondrial-only or cytosolic-only aaRSs, two genes, GARS and KARS, encode enzymes, glycyl-tRNA synthetase (GlyRS) and lysil-tRNA synthetase (LysRS), which catalyze the reaction in both mitochondria and cytosol.
Dominant GARS mutations have been found in patients with Charcot-Marie-Tooth disease type 2D (CMT2D; OMIM\#601472) and with distal hereditary motor neuronopathy type VA (HMN5A; OMIM\#600794) [48, 49]. Experimental evidence demonstrated a key role for glycine-tRNA synthetase in maintaining peripheral axons [50]. More recently a toxic role of mutant GARS on peripheral nerves has been shown [51]. The dominant transmission can be ascribed to either loss of function (haploinsufficiency) or a dominant-negative loss-of-function effect, but also aberrant pathogenic function of the mutant protein has been hypothesized [52]. Remarkably, most of the known mutations in cytoplasmic ARSs are associated with a CMT phenotype or related neuropathies. Mutations in YARS have been described in patients with Dominant Intermediate (DI) CMT type C [53], whereas AARS mutations have been found in patients with a CMT2 phenotype [54] and CMT2N [55]; HARS mutations have been also associated with axonal peripheral neuropathy [56].

Mutations in KARS have been initially described in a patient with an intermediate form of autosomal recessive Charcot-Marie-Tooth disease (CMTRIB; OMIM\#613641) [57], developmental delay, self-abusive behavior, dysmorphic features, and vestibular schwannoma. The p.Tyr173SerfsX7 variant represents a loss-of-function allele, and p.Leul33His represents a severely hypomorphic allele in yeast growth and aminoacylation assays, respectively. The presence of compound heterozygosity for a null and severely hypomorphic KARS allele may explain the more severe or complex neuropathy phenotype than those found associated with other heterozygous dominant-negative aaRS mutation. Very recently, KARS homozygous missense mutations have been identified by WES in affected subjects from three consanguineous Pakistani families, with nonsyndromic-hearingimpairment phenotype [58]. No auditory neuropathy and CMT were reported. It has been hypothesized that KARS variants affect aminoacylation by interfering with binding activity to tRNA and with formation of the active LysRS tetramer. Because of the proven localization of LysRS within the cochlea, the authors suggested that perturbations in aminoacylation might affect many of the cellular processes of the different specialized cells of the cochlea and therefore result in hearing impairment.

As GARS and KARS encode for both the mitochondrial and cytoplasmic aaRSs, the evaluation of mitochondrial involvement in these phenotypes is complicated and has not been studied in detail.

\section{Functional Studies}

Given the function of the mt-aaRSs, pathogenic mutations are expected to impair binding between tRNAs and their cognate amino acids, resulting in reduction of charged tRNA, albeit other defects can be envisaged, such as defective mitochondrial targeting. However, analysis of amino acylation is a tricky process that requires a large amount of starting material, and it is rarely performed. Usually the target of the studies is a downstream effect of mt-aaRSs dysfunction, 
such as altered mitochondrial protein synthesis or OXPHOS deficiency.

The lack of clear biochemical phenotypes (OXPHOS or mitochondrial protein synthesis defects) in skin fibroblasts and myoblasts from most of mutant aaRS2 patients prevented the use of cultured cells for functional studies. In few RARS2 patients, fibroblasts showed OXPHOS defects, with RC enzymes variably affected; YARS2 patients presented deficient mitochondrial protein synthesis in myotubes but not in cultured fibroblasts. Microscale oxygraphy (by Seahorse instrument) could be a more sensible approach to reveal biochemical defects in cell lines: in EARS2 mutant fibroblasts, microoxygraphic analysis revealed reduction of maximal respiratory rate and reduced oxygen consumption rate to extracellular acidification rate ratio, reflecting increased production of lactate [46]. Otherwise, induced pluripotent stem cells derived from patient cell lines, differentiated into various cell types, may offer advantages in future studies.

The high degree of conservation of several mt-aaRSs during evolution makes feasible the use of user-friendly bacterial and yeast model to evaluate the effects of identified variants on protein function. In some studies, the recombinant $\mathrm{mt}$-aaRSs proteins (i.e., mt-PheRS and mt-AspRS) were expressed and purified in Escherichia coli, allowing the comparison of enzyme parameters such as catalytic activity, ATP binding, and protein stability between mutant and wt species. In other works, (i.e., RARS2, HARS2, LARS2, and $K A R S$ ) mutations were studied on facultative aerobic yeast Saccharomyces cerevisiae. The yeast orthologous gene can be inactivated by homologous recombination and the resulting cells then transformed with a centromeric plasmid containing either the wild type or a mutant allele. Usually, all strains are able to grow in a medium containing a fermentable sugar, but strains with mutations affecting the OXPHOS system show decreased or abolished respiration and display a reduced growth on nonfermentable medium [31]. A restriction in the use of this approach is the high number of yeast aaRS genes coding enzymes with a double localization, both mitochondrial and cytosolic [59]. This limitation can be bypassed with a more complex strategy, based on the inactivation of the endogenous bifunctional enzyme and the reexpression of modified plasmids coding specifically either the cytosolic or the mitochondrial enzyme.

At the moment, no mouse model for mt-aaRSs has been fully characterized and published. Only two mutagenesisinduced mouse models for GARS have been described: a dominant model caused by an in-frame indel mutation at pro278 [52] and a second model with a point mutation (C201R) that leads to a nonconservative substitution [60]. The first model presented sensorimotor polyneuropathy with overt neuromuscular dysfunction and shortened life spans. Heterozygous mutant mice showed neurodegenerative changes at the neuromuscular junctions, whereas homozygous mutant mice were embryonic lethal. The dominant transmission was probably due to a gain of function [52]. The second model was less severe but displayed locomotor and sensory deficits, with myelination defects not detected in the former model, representing a valuable resource to study the mechanism of axonopathy resulting from GARS mutations [60].

The group of Aleksandra Trifunovic is currently dealing with DARS2 mouse models. They recently reported that constitutive Knock-Out (KO) mice had an early developmental arrest with embryonic lethality, whereas conditional cardiac and skeletal muscle KO mice showed dramatically shortened life span and a gradual increase in heart/body weight ratio, with strong reduction of all assembled respiratory chain complexes, except complex II, in both heart and skeletal muscle [61]. Two probably more interesting mouse models are undergoing study, based on different brain-specific DARS2 depletion in either adult forebrain neurons or in myelinproducing oligodendrocytes.

The production of conditional tissue-specific mouse models for different mt-aaRSs could provide an insight into the pathological mechanisms and the selective tissue involvement caused by mutations in these ubiquitously expressed enzymes.

\section{Discussion}

It is hard to explain why genetic defects in mt-aaRSs, which are ubiquitous enzymes necessary for mitochondrial translation, can cause so many different phenotypes usually affecting specific tissues or organs. Different hypotheses have been proposed to explain this variability, but none seems to cover all aspects of this phenomenon, that is probably the result of several different mechanisms altered in mt-aaRSs mutant patients.

The first patients with splice-site mutation in either DARS2 or RARS2 presented with encephalopathy. Differences in mRNA splicing machinery, in abundance of splicing factors, and splicing efficiency between neuronal and other cell types have been hypothesized to explain this tissue specificity. However, other patients harbouring DARS2 or RARS2 missense mutations have been reported later weakening the general value of this hypothesis. In addition to DARS2 and RARS2 mutations, also the tissue specificity present in HARS2 mutant patients may be influenced by splicing efficiency in different tissues/organs, in the latter case ovaries and ears. However, only one family has been described till now and the identification of other patients with HARS2 mutations is required to confirm this observation.

Other types of mutations and their location inside the protein could have an influence on the resulting phenotype. For instance, the AARS2 p.Arg592Trp mutation is located at the surface of the editing domain, which functions in deacylating the mischarged tRNAs, and is predicted to affect the recognition of mischarged tRNAs [38]. The pathogenic mechanism of this mutation may be linked to mitochondrial mistranslation and hence be different from other mt-aaRS defects. Why mistranslation would lead specifically to cardiomyopathy, while translation deficiencies caused by other mt-aaRSs mutations are usually not associated with heart damage remains an open question. Noteworthy, mutations affecting MTO1, an enzyme responsible for tRNA modifications that increases the accuracy and efficiency of mtDNA 
translation, have been found in patients with hypertrophic cardiomyopathy [62].

Alternatively, the bigger susceptibility of some cell types, in particular neurons, for aminoacyl-tRNA synthetase dysfunction could be linked to the variable expression of mitochondrial tRNAs in different cells, to the amount of cognate amino acid in a particular tissue [38], or to different threshold required for optimal enzymatic activity of each mt-aaRS. The brain expresses higher levels of mitochondrial-encoded tRNAs as compared to other examined tissue, including liver, vulva, testis and ovary, thymus, lymph node, and spleen [63]. Moreover, variations in the relative expression of tRNA isoacceptors among tissues have been observed: thus the tRNA levels could influence the usage of different codons among different tissues [63].

For AARS2 mutations, the tissue-specific manifestation of the cardiomyopathy could be explained by variable amino acid concentrations in different tissues; in fact, increased levels of alanine have been found in affected tissue (heart and muscle), but not in the unaffected liver. This observation could be due to a compensatory feedback mechanism occurring upon deficient tRNA ${ }^{\text {Ala }}$ aminoacylation or be an unspecific response to $\mathrm{RC}$ defect reflecting the alanine secreted from RC-deficient skeletal muscle for gluconeogenesis [38].

For some peculiar phenotypes, the pathological mechanisms seem to be specific and unique. In addition to YARS2 mutations, MLASA can be caused also by mutation in PUS1, encoding pseudouridylate synthase 1 . This enzyme is probably responsible for pseudouridylation of $\mathrm{tRNA}^{\mathrm{Tyr}}$. This observation suggests that tRNA ${ }^{\text {Tyr }}$ and not a general mitochondrial translation defect is specifically responsible for the combined myopathy and anemia in YARS2 mutant patients.

GARS and KARS, encoding enzymes acting both in mitochondria and cytosol, require ad hoc comments and considerations. Few pathological pictures, mainly CMT, have been described caused by defective cytoplasmic aaRSs; hence, the neuropathy phenotypes associated with GARS or KARS mutations have been ascribed mainly to impairment of the cytoplasmic enzyme. However, the possible contribution of faulty mitochondrial functionality in the development of the phenotype has not been fully analyzed. It should be noted that axonal CMT is frequently caused by mutations in a mitochondrial protein, MFN2, involved in mitochondrial fusion; however, it is not known if and how GlyRS or LysRS could be linked directly to mitochondrial dynamics or how a mitochondrial synthesis deficiency could lead to axonal dysfunction.

While mutations in GARS or other cytosolic aaRSs are inherited as dominant trait, KARS mutation are recessive and the presence of two mutant alleles has been suggested as responsible for the most severe phenotype in these patients, including acoustic neuroma and dysmorphisms besides CMT [57]. More recently, recessive KARS mutations have been reported in patients with nonsyndromic hearing impairment without neuropathy, expanding the spectrum of phenotypes associated with KARS mutation [58]. Unfortunately, the selective impairment of mitochondrial protein synthesis has not been investigated in these patients. Interestingly, hearing problems are present also in patients with PCH6 (due to RARS2 mutations) or Perrault syndrome (caused by LARS2 or HARS2 mutations). In addition, a number of different mutations in several genes of the mitochondrial genome, mainly MTRNR1 encoding the mitochondrial ribosomal RNA 12S, and many tRNA-encoding genes, are responsible for hearing impairment or deafness, in some cases associated with a variety of additional clinical features.

Mutations in DARS, encoding the cytosolic AspRS, have been recently found in a cohort of patients with a leukoencephalopathy characterized by hypomyelination with brain stem and spinal cord involvement and legs spasticity (HBSL, OMIM\#615281) [64]. HBSL resembles LBSL, which is caused by mutations in DARS2, suggesting that these two diseases might share a common underlying molecular pathology. Many aaRSs have additional functions beyond translation $[19,65]$ including AspRS, which is involved in asparagine biosynthesis [66]. It is possible that a common function for DARS and DARS2, not strictly related to aminoacylation, could be responsible for the very similar clinical presentations in HBSL and LBSL.

Increasing evidences suggest that mt-aaRSs have a role as "chaperone," besides their enzymatic function. According to the "channeling" hypothesis [15], interaction of mitochondrial tRNA with proteins, including mt-aaRSs, are not only necessary for tRNA synthesis, maturation, and function but also to protect tRNAs from degradation. Rapid turnover of free mt-tRNAs, not aminoacylated or bound to any protein partner, provides a general mechanism for the quality control of the tRNA pool within the human mitochondria. The data obtained from studies on mutated mt-tRNA support this hypothesis: pathogenic mutations in mt-tRNA are usually associated with a reduction in tRNA amount and a decrease of the aminoacylated form [15]. Similar indications came from studies on patients with aaRS2 mutations. In mutant RARS2 fibroblasts, the amount of tRNA ${ }^{\text {Arg }}$ transcript is low, but it is almost fully acylated. A residual ArgRS activity is probably present that could aminoacylate a small portion of the tRNAs ${ }^{A r g}$, whereas the uncharged tRNAs become unstable and are degraded [31]. In lymphocytes from patients bearing a homozygous SARS2 mutation, the amount of tRNA ${ }^{\text {Ser(AGY) }}$ was strongly reduced and fully nonacylated, whereas the amount of tRNA ${ }^{\operatorname{Ser}(\mathrm{UCN})}$ isoacceptor was nearly normal. The authors concluded that mutation in SARS2 significantly impairs the ability of the enzyme to aminoacylate selectively the tRNA ${ }^{\text {Ser(AGY)}}$, leading to degradation of the uncharged tRNA molecules [36]. Therefore, mutations affecting either $\mathrm{mt}$-tRNAs or $\mathrm{mt}$-aaRSs, perturbing their "stabilizing" interaction, lead to decreased tRNA levels.

Additional findings in support to the channeling theory have been obtained in both yeast and human cell lines $[15,67,68]$ : the overexpression of cognate, or even noncognate [69], mt-aaRSs has been demonstrated to be able to reduce/correct the deleterious effects of some mt-tRNA point mutations, leading to partial recovery of the steady-state levels of mutated tRNAs. Notably, the enzymatic activity of $\mathrm{mt}$-aaRSs seems to be not required for its rescuing effect. In 
yeast carrying tRNA mutations, the recovery has also been obtained by overexpression of an isolated C-terminal portion of yeast LeuRS, lacking the catalytic domain [70]; it has been proposed that this polypeptide may act as a chaperone, by directly interacting with mutant mt-tRNAs, making them less prone to degradation or maintaining them in a structural conformation fostered to react with the endogenous mtLeuRS.

\section{Conclusions}

Increasing evidences suggest that the pathophysiology of mtaaRSs-associated disorders is driven by processes and interactions that are not yet fully understood. For instance, tissuespecific proteins that could interact differentially with the wild-type or mutant mt-aaRSs, by inhibiting the enzymatic activity or any additional function, might be identified.

In general, mt-aaRSs are relatively poorly characterized enzymes but with growing importance in human mitochondrial medicine. Generation of animal models will allow us to better understand mt-aaRSs functions, interactions, and possibly tissue specificity. These studies will surely provide important knowledge about the pathogenetic role of synthetases in the different human diseases.

\section{Conflict of Interests}

The authors declare that there is no conflict of interests regarding the publication of this paper.

\section{References}

[1] R. W. Taylor and D. M. Turnbull, "Mitochondrial DNA mutations in human disease," Nature Reviews Genetics, vol. 6, no. 5, pp. 389-402, 2005.

[2] D. Ghezzi and M. Zeviani, "Assembly factors of human mitochondrial respiratory chain complexes: physiology and pathophysiology," Advances in Experimental Medicine and Biology, vol. 748, pp. 65-106, 2012.

[3] P. Smits, J. Smeitink, and L. van den Heuvel, "Mitochondrial translation and beyond: processes implicated in combined oxidative phosphorylation deficiencies," Journal of Biomedicine and Biotechnology, vol. 2010, Article ID 737385, 24 pages, 2010.

[4] K. Watanabe, "Unique features of animal mitochondrial translation systems: the non-universal genetic code, unusual features of the translational apparatus and their relevance to human mitochondrial diseases," Proceedings of the Japan Academy Series B, vol. 86, no. 1, pp. 11-39, 2010.

[5] M. Liu and L. Spremulli, "Interaction of mammalian mitochondrial ribosomes with the inner membrane," Journal of Biological Chemistry, vol. 275, no. 38, pp. 29400-29406, 2000.

[6] V. Ramakrishnan, "Ribosome structure and the mechanism of translation," Cell, vol. 108, no. 4, pp. 557-572, 2002.

[7] A. Marintchev and G. Wagner, "Translation initiation: structures, mechanisms and evolution," Quarterly Reviews of Biophysics, vol. 37, no. 3-4, pp. 197-284, 2004.

[8] G. Bertram, S. Innes, O. Minella, J. P. Richardson, and I. Stansfield, "Endless possibilities: translation termination and stop codon recognition," Microbiology, vol. 147, no. 2, pp. 255269, 2001.
[9] A. Zeharia, A. Shaag, O. Pappo et al., "Acute infantile liver failure due to mutations in the TRMU gene," American Journal of Human Genetics, vol. 85, no. 3, pp. 401-407, 2009.

[10] D. Ghezzi, E. Baruffini, T. B. Haack et al., "Mutations of the mitochondrial-tRNA modifier MTO1 cause hypertrophic cardiomyopathy and lactic acidosis," The American Journal of Human Genetics, vol. 90, pp. 1079-1087, 2012.

[11] J. A. M. Smeitink, O. Elpeleg, H. Antonicka et al., "Distinct clinical phenotypes associated with a mutation in the mitochondrial translation elongation factor EFTs," American Journal of Human Genetics, vol. 79, no. 5, pp. 869-877, 2006.

[12] L. Valente, V. Tiranti, R. M. Marsano et al., "Infantile encephalopathy and defective mitochondrial DNA translation in patients with mutations of mitochondrial elongation factors EFG1 and EFTu," American Journal of Human Genetics, vol. 80, no. 1, pp. 44-58, 2007.

[13] M. Ibba and D. Soll, "Aminoacyl-tRNA synthesis," Annual Review of Biochemistry, vol. 69, pp. 617-650, 2000.

[14] A. Nagao, T. Suzuki, T. Katoh, Y. Sakaguchi, and T. Suzuki, "Biogenesis of glutaminyl-mt tRNAGln in human mitochondria," Proceedings of the National Academy of Sciences of the United States of America, vol. 106, no. 38, pp. 16209-16214, 2009.

[15] R. Li and M.-X. Guan, "Human mitochondrial leucyl-tRNA synthetase corrects mitochondrial dysfunctions due to the tRNALeu(UUR) A3243G mutation, associated with mitochondrial encephalomyopathy, lactic acidosis, and stroke-like symptoms and diabetes," Molecular and Cellular Biology, vol. 30, no. 9, pp. 2147-2154, 2010.

[16] M. P. King and G. Attardi, "Post-transcriptional regulation of the steady-state levels of mitochondrial tRNAs in HeLa cells," Journal of Biological Chemistry, vol. 268, no. 14, pp. 10228-10237, 1993.

[17] K. Beebe, M. Mock, E. Merriman, and P. Schimmel, "Distinct domains of tRNA synthetase recognize the same base pair," Nature, vol. 451, no. 7174, pp. 90-93, 2008.

[18] L. Bonnefond, A. Fender, J. Rudinger-Thirion, R. Giegé, C. Florentz, and M. Sissler, "Toward the full set of human mitochondrial aminoacyl-tRNA synthetases: characterization of AspRS and TyrRS," Biochemistry, vol. 44, no. 12, pp. 48054816, 2005.

[19] E. V. Smirnova, V. A. Lakunina, I. Tarassov, I. A. Krasheninnikov, and P. A. Kamenski, "Noncanonical functions of aminoacyl-tRNA synthetases," Biochemistry, vol. 77, no. 1, pp. $15-25,2012$.

[20] S. Konovalova and H. Tyynismaa, "Mitochondrial aminoacyltRNA synthetases in human disease," Molecular Genetics and Metabolism, vol. 108, no. 4, pp. 206-211, 2013.

[21] G. C. Scheper, T. van der Klok, R. J. van Andel et al., "Mitochondrial aspartyl-tRNA synthetase deficiency causes leukoencephalopathy with brain stem and spinal cord involvement and lactate elevation," Nature Genetics, vol. 39, no. 4, pp. 534-539, 2007.

[22] L. Van Berge, S. Dooves, C. G. M. Van Berkel, E. Polder, M. S. Van Der Knaap, and G. C. Scheper, "Leukoencephalopathy with brain stem and spinal cord involvement and lactate elevation is associated with cell-type-dependent splicing of mtAspRS mRNA," Biochemical Journal, vol. 441, no. 3, pp. 955-962, 2012.

[23] P. Labauge, I. Dorboz, E. Eymard-Pierre, O. Dereeper, and O. Boespflug-Tanguy, "Clinically asymptomatic adult patient with extensive LBSL MRI pattern and DARS2 mutations," Journal of Neurology, vol. 258, no. 2, pp. 335-337, 2011. 
[24] S. Sharma, N. Sankhyan, A. Kumar, G. C. Scheper, M. S. Van Der Knaap, and S. Gulati, "Leukoencephalopathy with brain stem and spinal cord involvement and high lactate: a genetically proven case without elevated white matter lactate," Journal of Child Neurology, vol. 26, no. 6, pp. 773-776, 2011.

[25] P. Isohanni, T. Linnankivi, J. Buzkova et al., "DARS2 mutations in mitochondrial leucoencephalopathy and multiple sclerosis," Journal of Medical Genetics, vol. 47, no. 1, pp. 66-70, 2010.

[26] N. Miyake, S. Yamashita, K. Kurosawa et al., "A novel homozygous mutation of DARS2 may cause a severe LBSL variant," Clinical Genetics, vol. 80, no. 3, pp. 293-296, 2011.

[27] S. Yamashita, N. Miyake, N. Matsumoto et al., "Neuropathology of leukoencephalopathy with brainstem and spinal cord involvement and high lactate caused by a homozygous mutation of DARS2," Brain and Development, vol. 35, no. 4, pp. 312-316, 2013.

[28] M. Synofzik, J. Schicks, T. Lindig et al., "Acetazolamideresponsive exercise-induced episodic ataxia associated with a novel homozygous DARS2 mutation," Journal of Medical Genetics, vol. 48, no. 10, pp. 713-715, 2011.

[29] S. Edvardson, A. Shaag, O. Kolesnikova et al., "Deleterious mutation in the mitochondrial arginyl-transfer RNA synthetase gene is associated with pontocerebellar hypoplasia," American Journal of Human Genetics, vol. 81, no. 4, pp. 857-862, 2007.

[30] J. Rankin, R. Brown, W. B. Dobyns et al., "Pontocerebellar hypoplasia type 6: a British case with PEHO-like features," American Journal of Medical Genetics, Part A, vol. 152, no. 8, pp. 2079-2084, 2010.

[31] D. Cassandrini, M. R. Cilio, M. Bianchi et al., "Pontocerebellar hypoplasia type 6 caused by mutations in RARS2: definition of the clinical spectrum and molecular findings in five patients," Journal of Inherited Metabolic Disease, vol. 36, no. 1, pp. 43-53, 2012.

[32] Y. Namavar, P. G. Barth, P. R. Kasher et al., "Clinical, neuroradiological and genetic findings in pontocerebellar hypoplasia," Brain, vol. 134, no. 1, pp. 143-156, 2011.

[33] L. G. Riley, S. Cooper, P. Hickey et al., "Mutation of the mitochondrial tyrosyl-tRNA synthetase gene, YARS2, causes myopathy, lactic acidosis, and sideroblastic anemia - MLASA syndrome," American Journal of Human Genetics, vol. 87, no. 1, pp. 52-59, 2010.

[34] R. Shahni, Y. Wedatilake, M. A. Cleary, K. J. Lindley, K. R. Sibson, and S. Rahman, "A distinct mitochondrial myopathy, lactic acidosis and sideroblastic anemia (MLASA) phenotype associates with YARS2 mutations," American Journal of Medical Genetics Part A, vol. 161, no. 9, pp. 2334-2338, 2013.

[35] F. Sasarman, T. Nishimura, I. Thiffault, and E. A. Shoubridge, "A novel mutation in YARS2 causes myopathy with lactic acidosis and sideroblastic anemia," Human Mutation, vol. 33, no. 8, pp. 1201-1206, 2012.

[36] R. Belostotsky, E. Ben-Shalom, C. Rinat et al., "Mutations in the mitochondrial Seryl-tRNA synthetase cause hyperuricemia, pulmonary hypertension, renal failure in infancy and alkalosis, HUPRA syndrome," American Journal of Human Genetics, vol. 88, no. 2, pp. 193-200, 2011.

[37] H. Rivera, E. Martin-Hernandez, A. Delmiro et al., "A new mutation in the gene encoding mitochondrial seryl-tRNA synthetase as a cause of HUPRA syndrome," BMC Nephrology, vol. 13, no. 14, p. 195, 2013.

[38] A. Götz, H. Tyynismaa, L. Euro et al., "Exome sequencing identifies mitochondrial alanyl-tRNA synthetase mutations in infantile mitochondrial cardiomyopathy," American Journal of Human Genetics, vol. 88, no. 5, pp. 635-642, 2011.

[39] D. Diodato, L. Melchionda, T. B. Haack et al., "Mutations in mitochondrial aminoacyl tRNA synthetases identified by exome-sequencing," European Journal of Human Genetics, vol. 21, supplement 2, p. 244, 2013.

[40] V. Bayat, I. Thiffault, M. Jaiswal et al., "Mutations in the mitochondrial methionyl-tRNA synthetase cause a neurodegenerative phenotype in flies and a recessive ataxia (ARSAL) in humans," PLoS Biology, vol. 10, no. 3, Article ID e1001288, 2012.

[41] S. B. Pierce, K. M. Chisholm, E. D. Lynch et al., "Mutations in mitochondrial histidyl tRNA synthetase HARS2 cause ovarian dysgenesis and sensorineural hearing loss of Perrault syndrome," Proceedings of the National Academy of Sciences of the United States of America, vol. 108, no. 16, pp. 6543-6548, 2011.

[42] S. B. Pierce, K. Gersak, R. Michaelson-Cohen et al., "Mutations in LARS2, encoding mitochondrial leucyl-tRNA synthetase, lead to premature ovarian failure and hearing loss in Perrault syndrome," The American Journal of Human Genetics, vol. 92, pp. 614-620, 2013.

[43] H. E. Shamseldin, M. Alshammari, T. Al-Sheddi et al., "Genomic analysis of mitochondrial diseases in a consanguineous population reveals novel candidate disease genes," Journal of Medical Genetics, vol. 49, pp. 234-241, 2012.

[44] J. M. Elo, S. S. Yadavalli, L. Euro et al., "Mitochondrial phenylalanyl-tRNA synthetase mutations underlie fatal infantile Alpers encephalopathy," Human Molecular Genetics, vol. 21, pp. 4521-4529, 2012.

[45] A. Almalki, C. L. Alston, A. Parker et al., "Mutation of the human mitochondrial phenylalanine-tRNA synthetase causes infantile-onset epilepsy and cytochrome c oxidase deficiency," Biochim Biophys Acta, vol. 1842, no. 1, pp. 56-64, 2013.

[46] M. E. Steenweg, D. Ghezzi, T. Haack et al., "Leukoencephalopathy with thalamus and brainstem involvement and high lactate "LTBL" caused by EARS2 mutations," Brain, vol. 135, no. 5, pp. 1387-1394, 2012.

[47] B. Talim, A. Pyle, H. Griffin et al., "Multisystem fatal infantile disease caused by a novel homozygous EARS2 mutation," Brain, vol. 136, part 2, p. e228, 2012.

[48] A. Antonellis, R. E. Ellsworth, N. Sambuughin et al., "Glycyl tRNA synthetase mutations in Charcot-Marie-Tooth disease type 2D and distal spinal muscular atrophy type V," American Journal of Human Genetics, vol. 72, no. 5, pp. 1293-1299, 2003.

[49] R. Del Bo, F. Locatelli, S. Corti et al., "Coexistence of CMT-2D and distal SMA-V phenotypes in an Italian family with a GARS gene mutation," Neurology, vol. 66, no. 5, pp. 752-754, 2006.

[50] A. Antonellis, S.-Q. Lee-Lin, A. Wasterlain et al., "Functional analyses of glycyl-tRNA synthetase mutations suggest a key role for tRNA-charging enzymes in peripheral axons," Journal of Neuroscience, vol. 26, no. 41, pp. 10397-10406, 2006.

[51] W. W. Motley, K. L. Seburn, M. H. Nawaz et al., "Charcotmarie-tooth-linked mutant GARS is toxic to peripheral neurons independent of wild-type GARS levels," PLoS Genetics, vol. 7, no. 12, Article ID e1002399, 2011.

[52] K. L. Seburn, L. A. Nangle, G. A. Cox, P. Schimmel, and R. W. Burgess, "An active dominant mutation of glycyl-tRNA synthetase causes neuropathy in a Charcot-Marie-Tooth 2D mouse model," Neuron, vol. 51, no. 6, pp. 715-726, 2006.

[53] A. Jordanova, J. Irobi, F. P. Thomas et al., "Disrupted function and axonal distribution of mutant tyrosyl-tRNA synthetase in dominant intermediate Charcot-Marie-Tooth neuropathy," Nature Genetics, vol. 38, no. 2, pp. 197-202, 2006. 
[54] P. Latour, C. Thauvin-Robinet, C. Baudelet-Méry et al., "A major determinant for binding and aminoacylation of tRNA ${ }^{\text {Ala }}$ in cytoplasmic Alanyl-tRNA synthetase is mutated in dominant axonal Charcot-Marie-Tooth disease," American Journal of Human Genetics, vol. 86, no. 1, pp. 77-82, 2010.

[55] H. M. McLaughlin, R. Sakaguchi, W. Giblin et al., "A recurrent loss-of-function alanyl-tRNA synthetase (AARS) mutation in patients with charcot-marie-tooth disease type 2N (CMT2N)," Human Mutation, vol. 33, no. 1, pp. 244-253, 2012.

[56] A. Vester, G. Velez-Ruiz, H. M. McLaughlin et al., "A lossof-function variant in the human histidyl-tRNA synthetase (HARS) gene is neurotoxic in vivo," Human Mutation, vol. 34, pp. 191-199, 2013.

[57] H. M. McLaughlin, R. Sakaguchi, C. Liu et al., "Compound heterozygosity for loss-of-function lysyl-tRNA synthetase mutations in a patient with peripheral neuropathy," American Journal of Human Genetics, vol. 87, no. 4, pp. 560-566, 2010.

[58] R. L. P. Santos-Cortez, K. Lee, Z. Azeem et al., "Mutations in KARS, encoding lysyl-tRNA synthetase, cause autosomalrecessive nonsyndromic hearing impairment DFNB89," The American Journal of Human Genetics, vol. 93, pp. 132-140, 2013.

[59] K.-J. Chang, G. Lin, L.-C. Men, and C.-C. Wang, "Redundancy of non-AUG initiators: a clever mechanism to enhance the efficiency of translation in yeast," Journal of Biological Chemistry, vol. 281, no. 12, pp. 7775-7783, 2006.

[60] F. Achilli, V. Bros-Facer, H. P. Williams et al., "An ENU-induced mutation in mouse glycyl-tRNA synthetase (GARS) causes peripheral sensory and motor phenotypes creating a model of Charcot-Marie-Tooth type 2D peripheral neuropathy," DMM Disease Models and Mechanisms, vol. 2, no. 7-8, pp. 359-373, 2009.

[61] S. A. Dogan, C. Pujol, and A. Trifunovic, "Mitochondrial aspartyl-tRNA synthetase (DARS2) deficiency in mice," Experimental Gerontology, vol. 48, p. 696, 2013.

[62] E. Baruffini, C. Dallabona, F. Invernizzi et al., "MTO1 mutations are associated with hypertrophic cardiomyopathy and lactic acidosis and cause respiratory chain deficiency in humans and yeast," Human Mutation, vol. 34, no. 11, pp. 1501-1509, 2013.

[63] K. A. Dittmar, J. M. Goodenbour, and T. Pan, "Tissue-specific differences in human transfer RNA expression," PLoS Genetics, vol. 2, no. 12, pp. 2107-2115, 2006.

[64] R. J. Taft, A. Vanderver, R. J. Leventer et al., "Mutations in DARS cause hypomyelination with brain stem and spinal cord involvement and leg spasticity," The American Journal of Human Genetics, vol. 92, pp. 774-780, 2013.

[65] R. L. Hurto, "Unexpected functions of tRNA and tRNA processing enzymes," Advances in Experimental Medicine and Biology, vol. 722, pp. 137-155, 2011.

[66] L. Klipcan and M. Safro, "Amino acid biogenesis, evolution of the genetic code and aminoacyl-tRNA synthetases," Journal of Theoretical Biology, vol. 228, no. 3, pp. 389-396, 2004.

[67] C. De Luca, Y. Zhou, A. Montanari et al., "Can yeast be used to study mitochondrial diseases? Biolistic tRNA mutants for the analysis of mechanisms and suppressors," Mitochondrion, vol. 9, no. 6, pp. 408-417, 2009.

[68] S. G. Park, P. Schimmel, and S. Kim, "Aminoacyl tRNA synthetases and their connections to disease," Proceedings of the National Academy of Sciences of the United States of America, vol. 105, no. 32, pp. 11043-11049, 2008.

[69] A. Montanari, C. De Luca, L. Frontali, and S. Francisci, "Aminoacyl-tRNA synthetases are multivalent suppressors of defects due to human equivalent mutations in yeast mt tRNA genes," Biochimica et Biophysica Acta, vol. 1803, no. 9, pp. 10501057, 2010.

[70] S. Francisci, A. Montanari, C. De Luca, and L. Frontali, "Peptides from aminoacyl-tRNA synthetases can cure the defects due to mutations in mt tRNA genes," Mitochondrion, vol. 11, no. 6, pp. 919-923, 2011. 

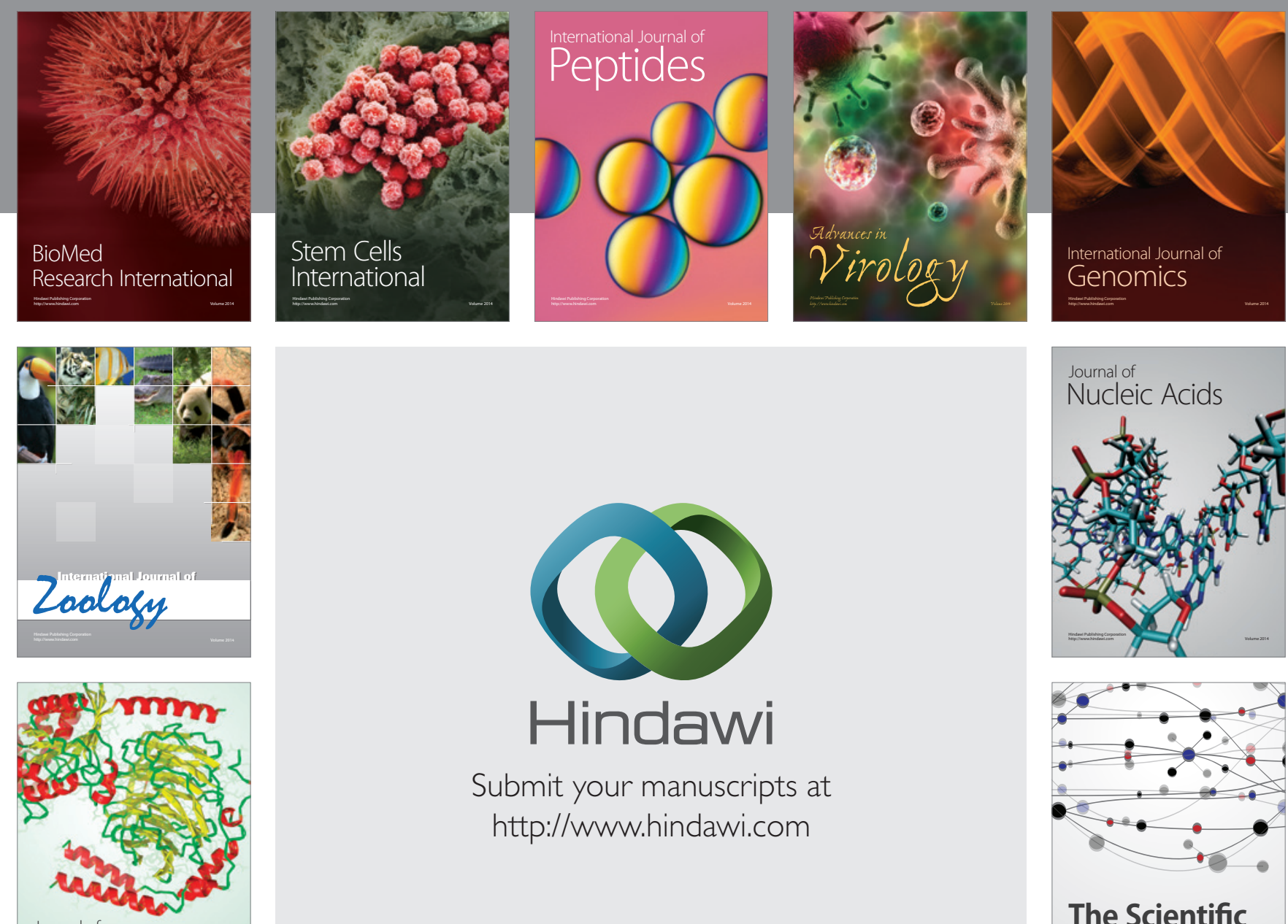

Submit your manuscripts at

http://www.hindawi.com

Journal of
Signal Transduction
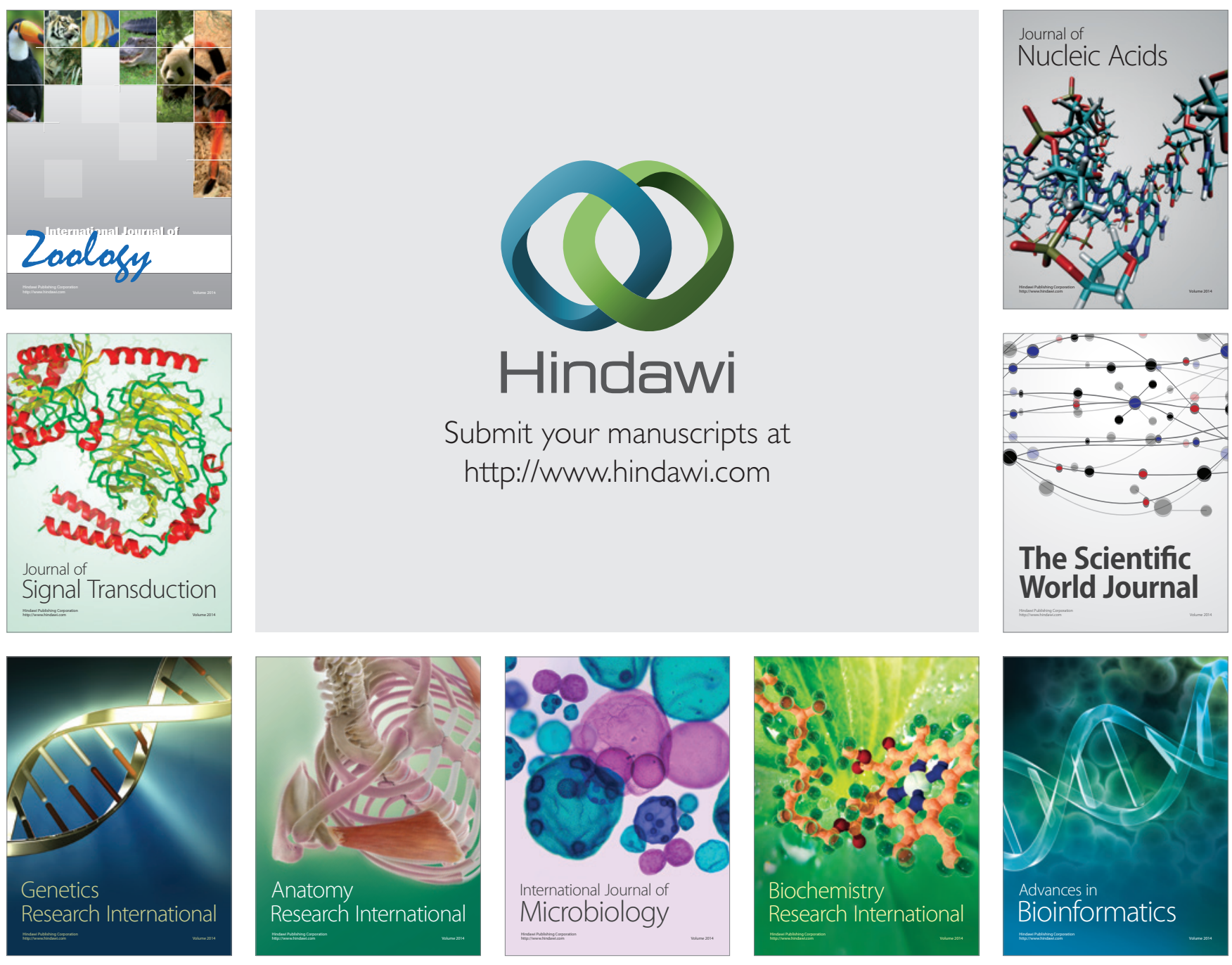

The Scientific World Journal
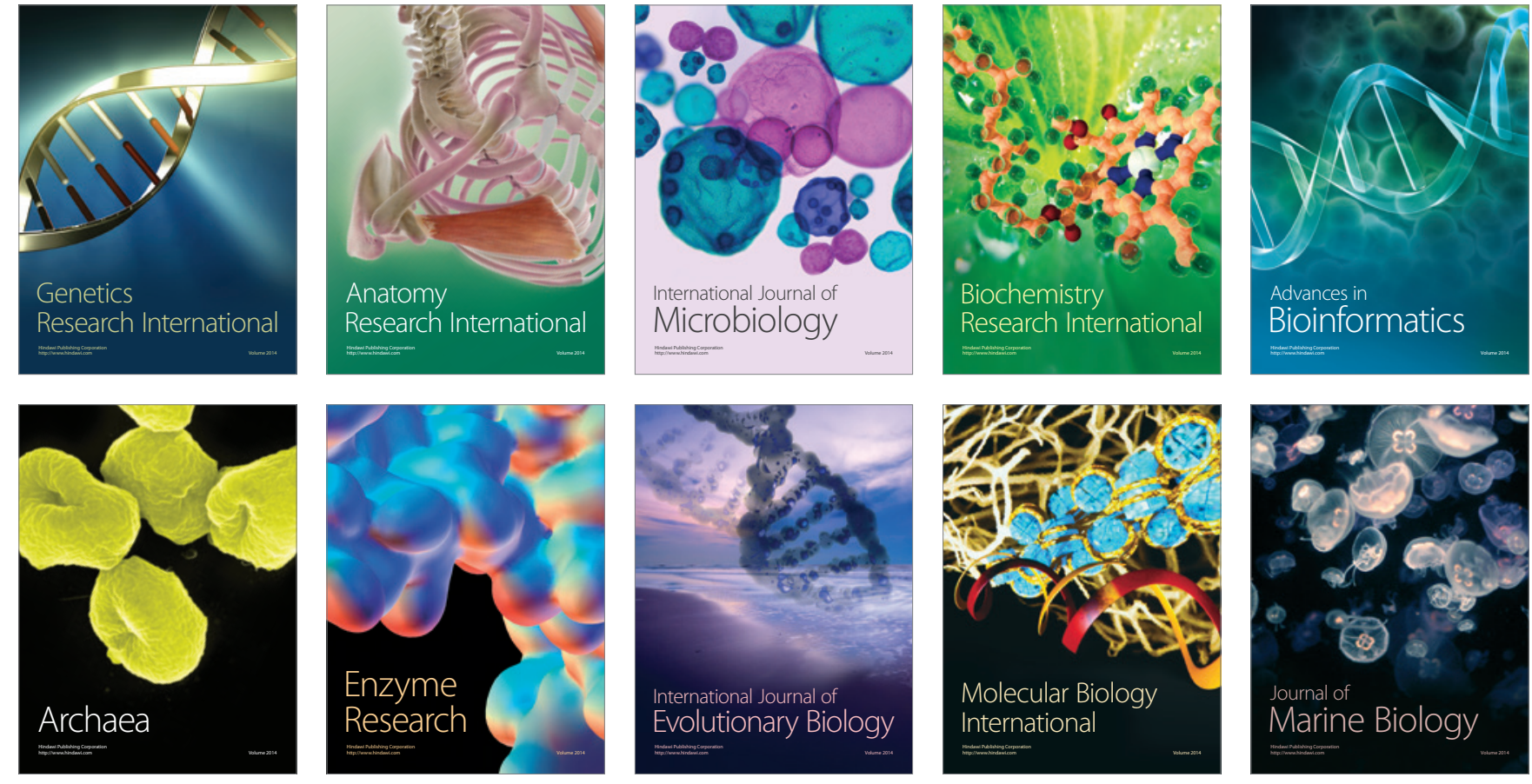